\title{
Immobilized Biocatalysts
}

Saccharomyces Yeasts

Wastewater Treatment

\author{
S. Fukui, A. Tanaka
}

Application of Biocatalysts

Immobilized by Prepolymer Methods

T. Kamihara, I. Nakamura

Regulation of Respiration and Its

Related Metabolism by Vitamin $\mathrm{B}_{1}$ and

Vitamin $\mathrm{B}_{6}$ in Saccharomyces Yeasts

H. Sahm

Anaerobic Wastewater Treatment

R. Sudo, S. Aiba

Role and Function of Protozoa in the Biological Treatment of Polluted Waters 

Immobilized Biocatalysts

Saccharomyces Yeasts

Wastewater Treatment 



\section{Immobilized Biocatalysts Saccharomyces Yeasts Wastewater Treatment}

Managing Editor: A. Fiechter

with 73 Figures and 45 Tables

Akademie-Verlag · Berlin 1984 
Die Originalausgabe erscheint im Springer-Verlag

Berlin-Heidelberg-New York-Tokyo

als Volume 29 der Schriftenreihe Advances in

Biochemical Engineering/Biotechnology

Vertrieb ausschließlich für die DDR und die sozialistischen Länder

Alle Rechte vorbehalten

(C) Springer-Verlag Berlin-Heidelberg 1984

Erschienen im Akademie-Verlag Berlin, DDR-1086 Berlin, Leipziger Straße 3-4

Lizenznummer: $202 \cdot 100 / 509 / 84$

Printed in the German Democratic Republic

Gesamtherstellung: VEB Druckerei „Thomas Müntzer“, 5820 Bad Langensalza

Umschlaggestaltung: Karl Salzbrunn

LSV 1315

Bestellnummer: 7633550 (6829)

07800 


\section{Managing Editor \\ Professor Dr. A. Fiechter \\ Institut für Biotechnologie \\ Eidgenössische Technische Hochschule, \\ Hönggerberg, \\ CH-8093 Zürich}

\section{Editorial Board}

Prof. Dr. S. Aiba

Prof. Dr. B. Atkinson

Prof. Dr. E. Bylinkina

Prof. Dr. Ch. L. Cooney

Prof. Dr. H. Dellweg

Prof. Dr. A. L. Demain

Prof. Dr. S. Fukui

Prof. Dr. K. Kieslich

Prof. Dr. R. M. Lafferty

Prof. Dr. K. Mosbach

Prof. Dr. H.J. Rehm

Prof. Dr. P. L. Rogers

Prof. Dr. H. Sahm

Prof. Dr. K. Schügerl

Prof. Dr. H. Suomalainen

Prof. Dr. S. Suzuki

Prof. Dr. H.Taguchi

Prof. Dr. G. T. Tsao
Department of Fermentation Technology, Faculty of Engineering, Osaka University, Yamada-Kami, SuitaShi, Osaka 565, Japan

University of Manchester, Dept. Chemical Engineering, Manchester/England

Head of Technology Dept., National Institute of Antibiotika. 3a Nagatinska Str., Moscow M-105/USSR Massachusetts Institute of Technology, Department of Chemical Engineering. Cambridge, Massachusetts 02139/USA

Techn. Universität Berlin, Lehrstuhl für Biotechnologie, Seestraße 13, D-1000 Berlin 65 Massachusetts Institute of Technology, Dept. of Nutrition \& Food Sc., Room 56-125, Cambridge, Mass. 02139/USA

Dept. of Industrial Chemistry, Faculty of Engineering, Sakyo-Ku, Kyoto 606, Japan Wissenschaftl. Direktor, Ges. für Biotechnolog. Forschung $\mathrm{mbH}$, Mascheroder Weg 1, D-3300 Braunschweig

Techn. Hochschule Graz, Institut für Biochem. Technol., Schlögelgasse 9, A-8010 Graz Biochemical Div., Chemical Center, University of Lund, S-22007 Lund/Sweden

Westf. Wilhelms Universität, Institut für Mikrobiologie, Tibusstraße 7-15, D-4400 Münster School of Biological Technology, The University of New South Wales. PO Box 1 ,

Kensington, New South Wales, Australia 2033

Institut für Biotechnologie, Kernforschungsanlage Jülich, D-5170 Jülich Institut für Technische Chemie, Universität Haṇnover, Callinstraße 3, D-3000 Hannover

Director, The Finnish State Alcohol Monopoly, Alko, P.O.B. 350, 00101 Helsinki 10/Finland

Tokyo Institute of Technology,

Nagatsuta Campus, Research Laboratory of Resources Utilization

4259, Nagatsuta, Midori-ku, Yokohama 227/Japan

Faculty of Engineering, Osaka University, Yamada-kami, Suita-shi, Osaka 565/Japan

Director, Lab. of Renewable Resources Eng., A. A. Potter Eng. Center, Purdue University, West Lafayette, IN $47907 /$ USA 
